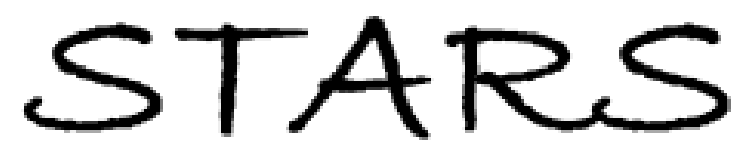

University of Central Florida

STARS

$1-1-1991$

\title{
Calculation Of The Base Current Components And Determination Of Their Relative Importance In Algaas/Gaas And Inalas/Ingaas Heterojunction Bipolar-Transistors
}

J. J. Liou

University of Central Florida

Find similar works at: https://stars.library.ucf.edu/facultybib1990

University of Central Florida Libraries http://library.ucf.edu

This Article is brought to you for free and open access by the Faculty Bibliography at STARS. It has been accepted for inclusion in Faculty Bibliography 1990s by an authorized administrator of STARS. For more information, please contact STARS@ucf.edu.

\section{Recommended Citation}

Liou, J. J., "Calculation Of The Base Current Components And Determination Of Their Relative Importance In Algaas/Gaas And Inalas/Ingaas Heterojunction Bipolar-Transistors" (1991). Faculty Bibliography 1990s. 272.

https://stars.library.ucf.edu/facultybib1990/272

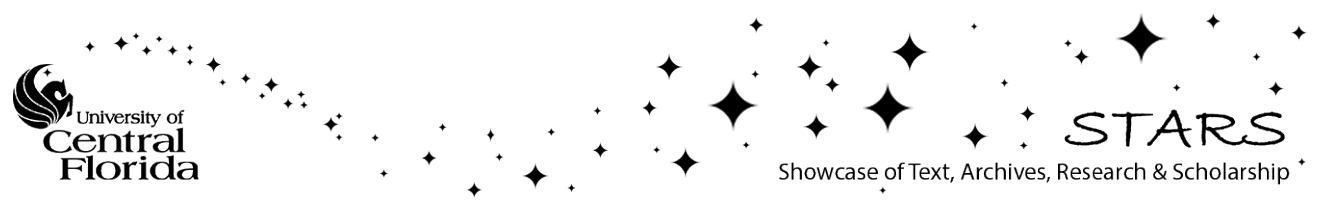




\section{Calculation of the base current components and determination of their relative importance in AIGaAs/GaAs and InAIAs/InGaAs heterojunction bipolar transistors}

Cite as: Journal of Applied Physics 69, 3328 (1991); https://doi.org/10.1063/1.348556 Submitted: 16 July 1990 . Accepted: 11 December 1990 . Published Online: 04 June 1998

J. J. Liou

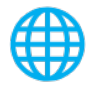

ARTICLES YOU MAY BE INTERESTED IN

Velocity saturation in few-layer $\mathrm{MoS}_{2}$ transistor

Applied Physics Letters 103, 233509 (2013); https://doi.org/10.1063/1.4840175

Emitter injection efficiency and base transport factor in InAs bipolar transistors Journal of Applied Physics 94, 5423 (2003); https://doi.org/10.1063/1.1606851

Tunneling in a finite superlattice

Applied Physics Letters 22, 562 (1973); https://doi.org/10.1063/1.1654509

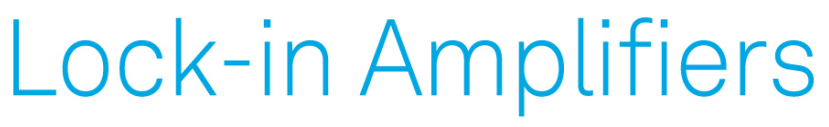
... and more, from DC to $600 \mathrm{MHz}$

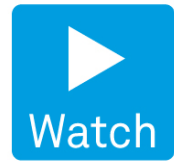




\title{
Calculation of the base current components and determination of their relative importance in AIGaAs/GaAs and InAIAs/InGaAs heterojunction bipolar transistors
}

\author{
J. J. Liou \\ Electrical Engineering Department, University of Central Florida, Orlando, Florida 32816
}

(Received 16 July 1990; accepted for publication 11 December 1990)

\begin{abstract}
The base current density $J_{B}$ is an important parameter in determining the common-emitter current gain $\beta$ of heterojunction bipolar transistors (HBTs). To develop an analytical $\beta$ model with which a circuit designer can quickly estimate the current gain in a HBT, it is also important to identify the dominant component of $J_{B}$ so that minimum computations are required. Based on heterojunction device physics, the three components of $J_{B}$ have been calculated, namely, the recombination current density in the base $J_{\mathrm{RB}}$, the recombination current density in the space-charge region $J_{\mathrm{SCR}}$, and the injection current density from the base to the emitter $J_{\mathrm{RE}}$, and have determined their relative importance to $J_{B}$ for abrupt $\mathrm{AlGaAs} / \mathrm{GaAs}$ and InAlAs/InGaAs HBTs under normal bias conditions. It is found that relative importance of the three current densities depends strongly on the bias condition, strongly on the density of states $N_{t I}$ at the emitter-base heterointerface, but weakly on the density of trapping states $N_{t B}$ in the bulk of the emitter-base space-charge region. Also, $J_{B}$ is relatively insensitive to device makeup such as doping concentration and layer thickness. Depending on $N_{t l}$ and on the bias condition, either $J_{\mathrm{SCR}}$ or $J_{\mathrm{RE}}$ is the dominant component for AlGaAs/GaAs HBTs and either $J_{\mathrm{SCR}}$ or $J_{\mathrm{RB}}$ is the dominant component for InAlAs/InGaAs HBTs. Effects of base and heterojunction grading on the present findings are also addressed.
\end{abstract}

\section{INTRODUCTION}

Heterojunction bipolar transistors (HBTs) can possess large common-emitter dc current gain $\beta^{1-3}$ when they operate in the forward-active mode. Many numerical studies on $\beta$ of HBTs exist in the literature, ${ }^{4,5}$ in which all components of the base current, e.g., the recombination current in the base, recombination current in the space-charge region, and hole injection from the base into the emitter, are included via the charge transport equation or detailed particle simulations such as the Monte Carlo simulation. In regard to modeling $\beta$ analytically, however, it is desirable to identify the dominant component of the base current so that a simple model providing physical intuition can be developed. Further, such a model allows HBT device designers to optimize $\beta$ performance by varying the HBT device makeup prior to the actual device fabrication. Nonetheless, uncertainties often arise to as which current component contributes most of the base current and thus contributes most to the degradation of $\beta$ in HBTs; a recent work $^{6}$ assumes that the base current stems mainly from electron-hole recombination current in the emitter-base heterojunction and hole injection from the $p$-type base into the $n$-type emitter while another ${ }^{7}$ believes that the decrease in the current gain is due mostly to recombination current in the quasineutral base region.

In this paper, we calculate all components of the base current density $J_{B}$ in InAlAs/InGaAs and AlGaAs/GaAs HBTs having abrupt junctions for a wide range of bias conditions and various device parameters. The resulting information should be useful to HBT designers to determine the relative importance of $J_{B}$ components and to quickly estimate the base current for predicting $\beta$ in HBTs. The effects of the junction of grading and base grading, which are two popular approaches to enhance the HBT performance, on the base current components will also be discussed.

\section{THEORY}

Consider an $n / p^{+} / n$ AlGaAs/GaAs or InAlAs/ InGaAs HBT (Fig. 1) under normal bias conditions (emitter-base applied voltage $V_{\mathrm{BE}}>0$ and base-collector applied voltage $V_{\mathrm{BC}}<0$ ). In Fig. 1, $X_{1}$ and $X_{2}$ are the edges of the emitter-base space-charge region and $X_{3}$ and $X_{4}$ are the edges of the base-collector space-charge region. The base current density $J_{B}$ consists of the following components: (i) recombination current density $J_{\mathrm{RB}}$ in the quasineutral base; (ii) recombination current density $J_{\mathrm{SCR}}$ in the emitter-base space-charge region, which includes both the recombination current density in the bulk of the space-charge region $\left(J_{\mathrm{SCRB}}\right)$ and the recombination current density at the heterointerface $\left(J_{\mathrm{SCRI}}\right)$; and (iii) hole current density $J_{\mathrm{RE}}$ injected from the base into the emitter. Surface recombination current density $J_{S}$ in the extrinsic base region is neglected in our treatment. According to a two-dimensional analysis by Hiraoka and Yashida, ${ }^{8} J_{S}$ is suppressed and is insignificant in a HBT having a very thin base. Thus the present one-dimensional treatment suffices for some HBTs with a base width less than or equal to about $1000 \AA$ (Ref. 8) or for HBTs with an emitter edge-thinning structure proposed by Lin and Lee. ${ }^{9}$ For other HBTs, however, $J_{S}$ is important and needs to be taken into account. 


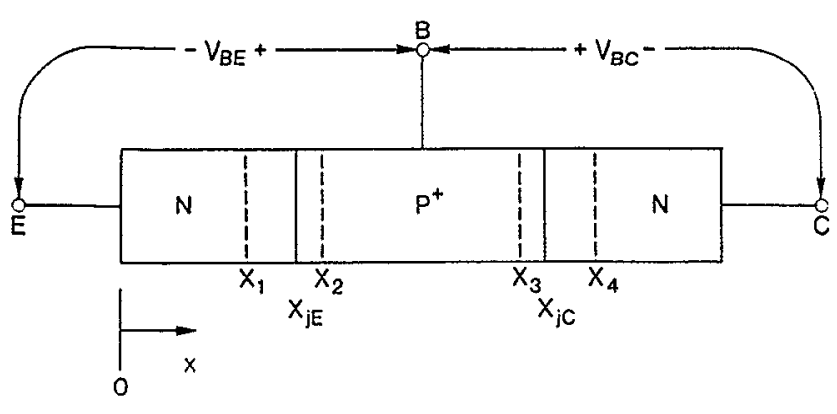

FIG. 1. Schematic illustration of an $n / p^{+} / n$ heterojunction bipolar transistor structure.

\section{A. $J_{\mathrm{RB}}$}

To model $J_{\mathrm{RB}}$, we employ a two-valley recombination model in which electrons in the base are assumed to reside predominately in $L$ and $\Gamma$ valleys, as evidenced from the Monte Carlo simulation by Rockett ${ }^{10}$ and from a selfconsistent particle simulation by Katoh and Kurata. ${ }^{1}$ Figure 2 shows schematically the electron currents in the base based on this model. In the figure, $J_{n}\left(X_{2}\right)$ is the electron current density injected from the emitter into the base and ${ }^{7}$

$$
\gamma=1 /\left(1+n_{\Gamma} / n_{L}\right),
$$

where $n_{L}$ is the electron density in the $L$ valley and $n_{\Gamma}$ is the electron density in the $\Gamma$ valley. Also ${ }^{7}$

$$
n_{\Gamma} / n_{L}=1 /\left[4\left(m_{L} / m_{\Gamma}\right)^{1.5} \exp \left(-\Delta E_{\Gamma-L} / k T\right)\right],
$$

where $m_{L}$ and $m_{\Gamma}$ are the effective masses for $L$ - and $\Gamma$-valley electrons, $\Delta E_{\Gamma-L}$ is the energy difference between $\Gamma$ and $L$ valleys, $k$ is Boltzmann constant, and $T$ is the absolute temperature. Also $\alpha_{L}$ and $\alpha_{\Gamma}$ are the base transport factors in $L$ valley and $\Gamma$ valley, respectively:

$$
\alpha_{L}=1 / \cosh \left[W_{B} /\left(D_{L} \tau_{L}\right)^{0.5}\right]
$$

and

$$
\alpha_{\Gamma}=1 / \cosh \left[W_{B} /\left(D_{\Gamma} \tau_{\Gamma}\right)^{0.5}\right],
$$

where $W_{B}$ is the quasineutral base width, $D_{L}$ and $D_{\Gamma}$ are the average electron diffusion coefficients in $L$ and $\Gamma$ valleys of the base, and $\tau_{L}$ and $\tau_{\Gamma}$ are the electron lifetimes in $L$ and $\Gamma$ valleys of the base. Note that $W_{B}=X_{3}-X_{2}$

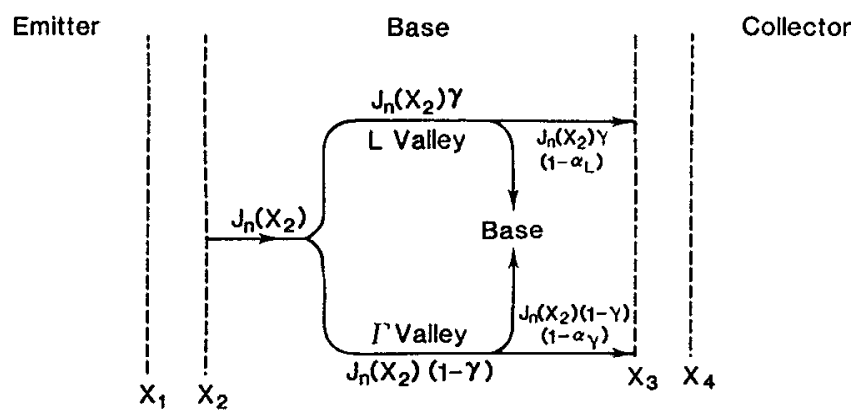

FIG. 2. Qualitative illustration of the recombination current in the base of the HBT based on two-the-valley recombination model. $\approx X_{j C}-X_{\mathrm{jE}}$ (Fig. 1) because the base region is doped heavier than the emitter and collector regions in HBTs.

Because of the presence of the conduction-band discontinuity (or spike) at the heterointerface $\left(x=X_{j E}\right)$ and the velocity overshoot phenomenon in the thin base region due to high electric field in the base-collector junction, the conventional drift-diffusion model is no longer applicable in describing the free-carrier injection from the emitter to the base in HBTs; ${ }^{3,4}$ instead, thermionic and tunneling become the dominant charge-transport mechanisms in the emitter-base space-charge region. ${ }^{3,4}$ Using the thermionictunneling model suggested in, ${ }^{3}$ we have

$J_{n}\left(X_{2}\right)=\left(q v_{n} / 4\right)\left[n\left(X_{j E}^{-}\right)-n\left(X_{j E}^{+}\right) \exp \left(-\Delta V_{d} / V_{T}\right)\right] \xi$,

where $V_{T}$ is the thermal voltage, $\xi$ is the electron tunneling coefficient defined in Ref. 3, and $\Delta V_{c}$ is the conductionband discontinuity at $X_{j E}$ (Refs. 12,13):

$$
\Delta V_{c}=\left|\chi_{E}-\chi_{B}\right|,
$$

where $\chi_{E}$ and $\chi_{B}$ are the electron affinity in the emitter and in the base. Also, ${ }^{3}$

$$
\begin{aligned}
& v_{n}=\left[8 k T /\left(\pi m_{n}\right)\right]^{0.5}, \\
& n\left(X_{j E}^{-}\right)=N_{E} \exp \left(-V_{B 1} / V_{T}\right), \\
& n\left(X_{j E}^{+}\right)=N_{E}\left(n_{i B} / n_{i E}\right) \exp \left[-\left(V_{B 1}+\Delta V_{i}\right) / V_{T}\right] .
\end{aligned}
$$

In the above equations, $m_{n} \approx 0.5\left(m_{L}+m_{\Gamma}\right)$ is the average effective electron mass, $N_{E}$ is the emitter doping concentration, $V_{B 1}$ is the base side of the junction barrier height, $n_{i E}$ and $n_{i B}$ are the emitter and base intrinsic carrier concentrations, and $\Delta V_{i}$ is the intrinsic Fermi potential discontinuity at $X_{j E^{\prime}}: 12,13$

$$
\begin{aligned}
V_{B 1}= & \left(V_{\mathrm{bi}}-V_{\mathrm{BE}}\right)\left\{1-\left[N_{E} \epsilon_{E} /\left(N_{E} \epsilon_{E}+N_{B} \epsilon_{B}\right)\right]\right\}, \\
\Delta V_{i}= & -\left|\chi_{E}-\chi_{B}\right|+(0.5 / q)\left|E_{\mathrm{GE}}-E_{\mathrm{GB}}\right| \\
& +0.5 V_{T} \ln \left(N_{\mathrm{CE}} N_{\mathrm{VB}} / N_{\mathrm{CB}} N_{\mathrm{VE}}\right) .
\end{aligned}
$$

$N_{B}$ is the base doping concentration, $\epsilon_{E}$ and $\epsilon_{B}$ are the emitter and base dielectric permittivities, $E_{\mathrm{GE}}$ and $E_{\mathrm{GB}}$ are the energy band gaps in the emitter and in the base, $N_{\mathrm{CE}}$ and $N_{\mathrm{CB}}$ are the effective density states in the conduction band of the emitter and the base, $N_{\mathrm{VE}}$ and $N_{\mathrm{VB}}$ are the effective density states in the valence band of the emitter and the base, and $V_{\mathrm{bi}}$ is the junction builtin potential: ${ }^{12,13}$

$$
\begin{aligned}
V_{\mathrm{bi}}= & \left|\chi_{E}-\chi_{B}\right|-(0.5 / q)\left|E_{\mathrm{GE}}-E_{\mathrm{GB}}\right| \\
& +V_{T} \operatorname{lr}\left(N_{E} N_{B} / n_{i E} n_{i B}\right) \\
& +0.5 V_{T} \ln \left(N_{\mathrm{CB}} N_{\mathrm{VE}} / N_{\mathrm{CE}} N_{\mathrm{VB}}\right) .
\end{aligned}
$$

Finally,

$$
J_{\mathrm{RB}}=J_{n}\left(X_{2}\right)\left[\gamma\left(1-\alpha_{L}\right)+(1-\gamma)\left(1-\alpha_{\Gamma}\right)\right] .
$$

\section{B. $J_{\mathrm{RE}}$}

Since the emitter of a HBT is usually very thin and lightly doped, the recombination current in the bulk of the 
TABLE I. Device and material parameters used for AlGaAs/GaAs HBTs (Refs. 3, 20, 22).

\begin{tabular}{|c|c|c|c|c|c|c|c|c|c|c|c|c|c|}
\hline $\begin{array}{c}\sigma \\
\left(\mathrm{cm}^{2}\right)\end{array}$ & $\begin{array}{c}N_{t B} \\
\left(\mathrm{~cm}^{-3}\right)\end{array}$ & $\begin{array}{c}N_{t I} \\
\left(\mathrm{~cm}^{-2}\right)\end{array}$ & $\begin{array}{c}\Delta E_{\Gamma-L} L \\
(\mathrm{eV})\end{array}$ & $\begin{array}{c}D_{L} \\
\left(\mathrm{~cm}^{2} / \mathrm{s}\right)\end{array}$ & $\begin{array}{c}D_{\mathrm{r}} \\
\left(\mathrm{cm}^{2} / \mathrm{s}\right)\end{array}$ & $\begin{array}{c}\tau_{L} \\
\text { (ps) }\end{array}$ & $\begin{array}{c}\tau_{\Gamma} \\
\text { (ps) }\end{array}$ & $m_{L} / m_{e}$ & $m_{\Gamma} / m_{e}$ & $\begin{array}{c}E_{\mathrm{GE}} \\
(\mathrm{eV})\end{array}$ & $\begin{array}{l}E_{\mathrm{GB}} \\
(\mathrm{eV})\end{array}$ & $\begin{array}{c}\chi_{E} \\
(\mathrm{eV})\end{array}$ & $\begin{array}{c}\chi_{B} \\
(\mathrm{eV})\end{array}$ \\
\hline $10^{-14}$ & $10^{14}$ & $10^{12}$ & 0.33 & 19 & 190 & 250 & 120 & 0.56 & 0.063 & 1.79 & 1.42 & 3.74 & 4.07 \\
\hline
\end{tabular}

quasineutral emitter can be assumed negligible. This, together with the diffusion-current-only assumption, leads to

$$
J_{\mathrm{RE}} \approx q D_{p} p\left(X_{\mathrm{I}}\right) / X_{1}
$$

where $D_{p}$ is the average hole diffusion coefficient in the emitter, $X_{1}$ can be calculated from the conventional depletion model, ${ }^{14}$ and, if the quasi-Fermi potentials are assumed flat in the space-charge region,

$p\left(X_{1}\right)=N_{B} \exp \left(n_{i E} / n_{i B}\right) \exp \left[-\left(V_{\mathrm{bi}}-V_{\mathrm{BE}}+\Delta V_{i}\right) / V_{T}\right]$.

\section{C. $J_{\mathrm{SCR}}$}

In homojunction bipolar transistors, $J_{\mathrm{SCR}}$ involves only the recombination current density $J_{S C R B}$ in the bulk of the space-charge region. For HBTs, however, the recombination current density $J_{\mathrm{SCRI}}$ at the heterojunction interface states is not negligible due to sizeable interface states resulting from lattice mismatch between the two different materials. ${ }^{15}$ Thus,

$$
J_{\mathrm{SCR}}=J_{\mathrm{SCRI}}+J_{\mathrm{SCRB}} \text {. }
$$

\section{1. $J_{S C R B}$}

The free-carrier recombination mechanism in the bulk of the space-charge region can be described by the Shockley-Read-Hall (SRH) model. ${ }^{6}$ The widely used and simplest SRH model is based on the assumption that there is only one type of trap that dominates the recombination process and that the traps are uniformly distributed in the middle of the energy band gap. ${ }^{17}$ From this, together with the assumption that the space-charge region resides entirely in the emitter side of the junction, we obtain the bulk recombination rate $U_{B}$ :

$$
U_{B}=\sigma v_{n} N_{t B} n_{i E}^{2}\left[\exp \left(V_{\mathrm{BE}} / V_{T}-1\right] /\left(n+p+2 n_{i E}\right),\right.
$$

where $\sigma$ is the capture cross section, $N_{t B}$ is the trapping density in the bulk of the space-charge region. Therefore

$$
J_{\mathrm{SCRB}}=q \int_{X_{1}}^{X_{2}} U_{B} d x \approx q \int_{X_{1}}^{X_{j E}} U_{B} d x .
$$

$U_{B}$ becomes $U_{B \max }$ when $n=p$ (Ref. 17):

$$
U_{B \max }=0.5 \sigma v_{n} N_{l B} n_{i E} \exp \left(V_{\mathrm{BE}} / 2 V_{T}\right) \text {. }
$$

The conventional approach is then to assume $n=p$ occurs in the entire space-charge region. This and Eq. (17) yield $J_{\mathrm{SCRB}} \approx q U_{B \max }\left(X_{j E}-X_{1}\right)$. This model is incorrect because, as pointed out recently by Shur, ${ }^{18} n=p$ does not occur in the entire space-charge region and thus $X_{j E}$ - $X_{1}$ in the model should be replaced by a width within which $U_{B \max }$ occurs. Using Taylor series to expand the function in Eq. (17), Shur more accurately obtains ${ }^{18}$

$$
\begin{aligned}
J_{\mathrm{SCRB}}= & q(\pi / 2)^{0.5} V_{T} n_{i E} \sigma v_{n} N_{l B}\left\{q N _ { E } \left[2 V_{T} \ln \left(N_{E} / n_{i E}\right)\right.\right. \\
& \left.\left.-V_{\mathrm{BE}}\right] / \epsilon_{B}\right\}^{-0.5} \exp \left(V_{\mathrm{BE}} / 2 V_{T}\right) .
\end{aligned}
$$

2. $J_{S C R I}$

Since interface states are arranged along $x=X_{j E}$ plane. ${ }^{15} J_{\text {SCRI }}$ can be expressed as ${ }^{19}$

$$
J_{\mathrm{SCRI}}=q \int_{X_{1}}^{X_{2}} U_{I} \delta\left(x-X_{j E}\right) d x=q U_{I},
$$

where $U_{I}$ is the SRH recombination rate at the interface states and $\delta$ is the delta function. Putting the condition $n \approx p$ at $x=X_{j E}$ into the SRH model yields ${ }^{19}$

$$
J_{\mathrm{SCRI}} \approx q U_{I \max }=0.5 q \sigma v_{n} N_{t I} n_{i E} \exp \left(V_{\mathrm{BE}} / 2 V_{T}\right),
$$

where $N_{t I}\left(\# / \mathrm{cm}^{2}\right)$ is the trapping density at the heterointerface.

\section{ILLUSTRATION AND DISCUSSION}

We consider both abrupt $\mathrm{Al}_{0.3} \mathrm{Ga}_{0.7} \mathrm{As} / \mathrm{GaAs}$ and $\mathrm{In}_{0.52} \mathrm{Al}_{0.48} \mathrm{As} / \mathrm{In}_{0.53} \mathrm{Ga}_{0.47} \mathrm{As}$ HBTs, which are prominent devices for microwave and electro-optics applications. Material parameters such as energy band gap, elcctron effective mass, and electron affinity for $\mathrm{Al}_{0.3} \mathrm{Ga}_{0.7} \mathrm{As} / \mathrm{GaAs}$ and $\mathrm{In}_{0.52} \mathrm{Al}_{0.48} \mathrm{As} / \mathrm{In}_{0.53} \mathrm{Ga}_{0.47} \mathrm{As}$ HBTs can be found in Refs. 20 and 21 and are given in Tables I and II, respectively.

Components of the base current density versus $V_{\mathrm{BE}}$ for

\begin{tabular}{|c|c|c|c|c|c|c|c|c|c|c|c|c|c|}
\hline $\begin{array}{c}\sigma \\
\left(\mathrm{cm}^{2}\right)\end{array}$ & $\begin{array}{c}N_{t B} \\
\left(\mathrm{~cm}^{-3}\right)\end{array}$ & $\begin{array}{c}N_{t I} \\
\left(\mathrm{~cm}^{-2}\right)\end{array}$ & $\begin{array}{c}\Delta E_{\Gamma-L} \\
(\mathrm{eV})\end{array}$ & $\begin{array}{c}D_{L} \\
\left(\mathrm{~cm}^{2} / \mathrm{s}\right)\end{array}$ & $\begin{array}{c}D_{\mathrm{r}} \\
\left(\mathrm{cm}^{2} / \mathrm{s}\right)\end{array}$ & $\begin{array}{c}\tau_{L} \\
(\mathrm{ps})\end{array}$ & $\begin{array}{c}\tau_{r} \\
\text { (ps) }\end{array}$ & $m_{L} / m_{e}$ & $m_{\Gamma} / m_{c}$ & $\begin{array}{l}E_{\mathrm{GE}} \\
(\mathrm{eV})\end{array}$ & $\begin{array}{l}E_{\mathrm{GB}} \\
(\mathrm{eV})\end{array}$ & $\begin{array}{c}\chi_{E} \\
(\mathrm{eV})\end{array}$ & $\begin{array}{c}X_{B} \\
(\mathrm{eV})\end{array}$ \\
\hline $10^{-14}$ & $10^{14}$ & $10^{12}$ & 0.5 & 4.0 & 40 & 250 & 120 & 0.2 & 0.045 & 1.47 & 0.77 & 4.38 & 4.88 \\
\hline
\end{tabular}
an $\mathrm{Al}_{0.3} \mathrm{Ga}_{0.7} \mathrm{As} / \mathrm{GaAs} \mathrm{HBT}$ and for an $\mathrm{In}_{0.52} \mathrm{Al}_{0.48} \mathrm{As} / \mathrm{In}_{0.53} \mathrm{Ga}_{0.47} \mathrm{As}$ HBT are plotted in Figs. 3 and 4 , respectively. Practical device parameters used for calculations for AlGaAs/GaAs and InAlAs/InGaAs HBTs $^{3,7,22}$ also appear in Tables I and II, respectively. Values of $N_{t B}$ and $N_{t I}$ chosen are usual for MBE-grown abrupt AlGaAs/GaAs heterojunctions. ${ }^{3,22}$ The HBTs have a typ-

TABLE II. Device and material parameters used for InAlAs/InGaAs HBTs (Refs. 7 and 21). 


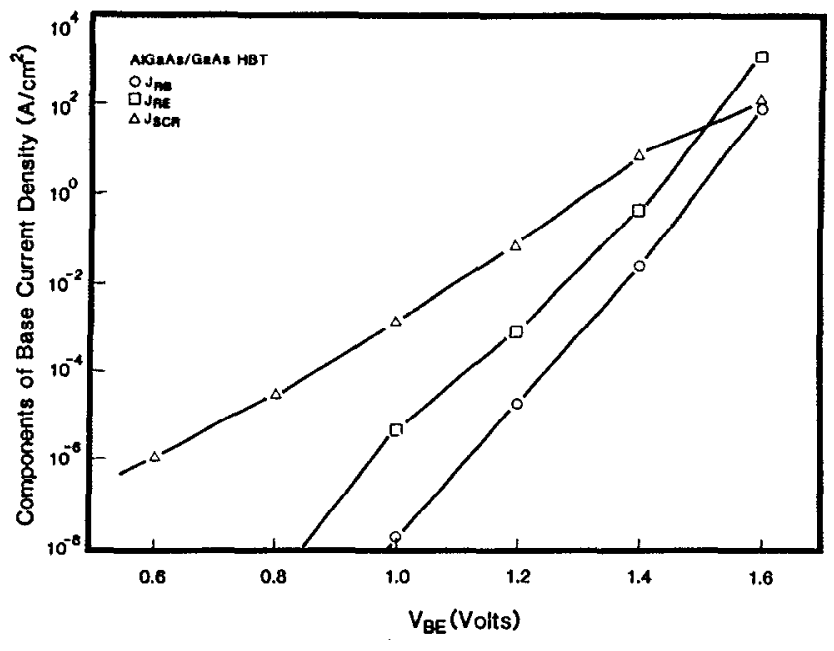

FIG. 3. Comparison of the components of the base current density vs the emitter-base applied voltage for an AlGaAs/GaAs HBT.

ical device makeup of $N_{E}=10^{17} \mathrm{~cm}^{-3}, N_{B}$ $=10^{19} \mathrm{~cm}^{-3}, X_{j E}=1000 \AA$, and $X_{j C}=2000 \AA$. As shown in Figs. 3 and 4, all components increase with increasing $V_{\mathrm{BE}}$, and $J_{\mathrm{SCR}}$ is the most important base-current component in both HBTs for small medium $V_{\mathrm{BE}}$. For larger $V_{\mathrm{BE}}, J_{\mathrm{RE}}$ and $J_{\mathrm{RB}}$ become the dominant component in AlGaAs/GaAs and InAlAs/InGaAs HBTs, respectively. In Figs. 5-14, the parameters in Tables I and II and the above device makeup are still used except that one of the parameters is varied. Also, $V_{\mathrm{BE}}$ is fixed at $1.4 \mathrm{~V}$, which is the voltage at which most HBT circuits are designed to operate. Figures 5 and 6 illustrate the dependencies of $J_{B}$ components on $W_{B}$. It is shown that $J_{\mathrm{RB}}$ increases slightly as $W_{B}$ increases, but this variation does not alter notably the trends shown in Figs. 3 and 4 regarding the relative importance of $J_{B}$ components. Figures 7 and 8 show the dependencies of $J_{\mathrm{RB}}$ and $J_{\mathrm{RE}}$ with respect to $N_{B}$. Variation

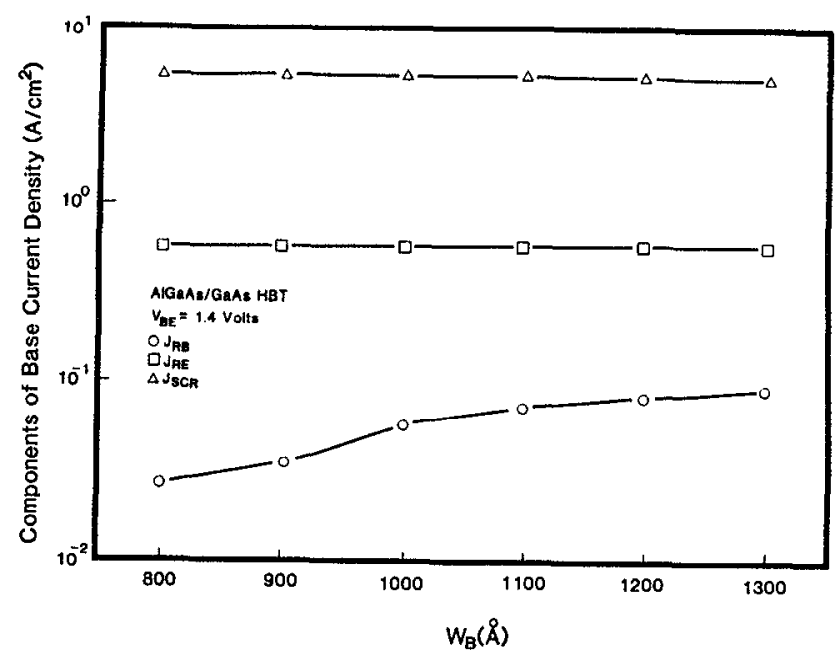

FIG. 5. Comparison of the components of the base current density vs the base width for an AlGaAs/GaAs HBT.

of $\tau$ alters moderately $J_{\mathrm{RB}}$ (Figs. 9 and 10) and changing $N_{t B}$ has no effect on $J_{\mathrm{SCR}}$ at this particular voltage (Figs. 11 and 12). However, $J_{\mathrm{SCR}}$ is very sensitive to $N_{t I}$ in both devices as shown in Figs. 13 and 14.

To assess the validity of the present analysis, data available in the literature ${ }^{23,24}$ are presented here to provide a comparison with the theoretical predications, as shown in Figs. 15 and 16 for an AlGaAs/GaAs HBT and an InAlAs/InGaAs HBT, respectively. The present model predicts fairly accurately the total base current density of the $\mathrm{AlGaAs} / \mathrm{GaAs} \mathrm{HBT}$, but discrepancies are found between the present model and measurement for the InAlAs/ InGaAs HBT. When the InAlAs/InGaAs HBT is subject to large forward voltages, the model overestimates the base current density by at most a factor of about 10 . This may be attributed to high-level injection and the non-negligible voltage drops in the quasineutral regions of the HBT at

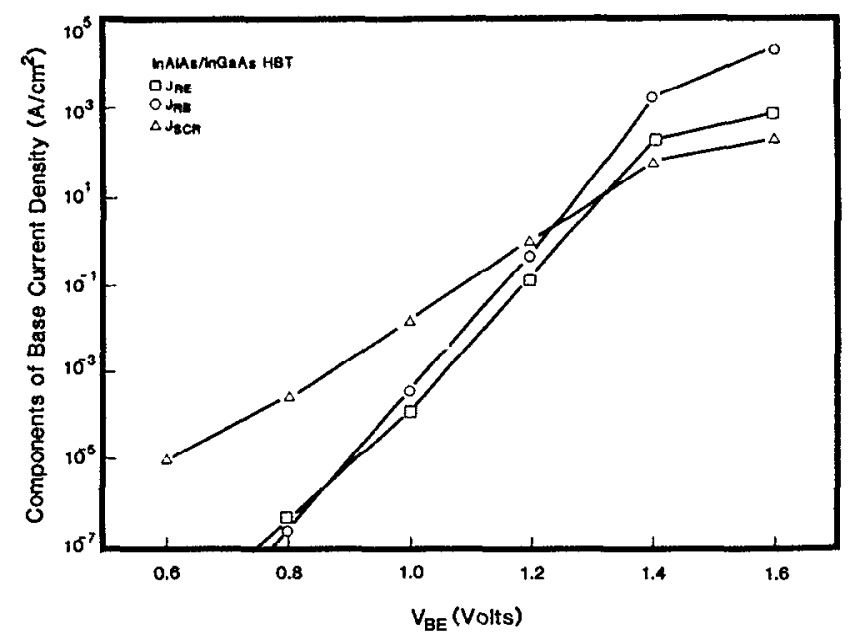

FIG. 4. Comparison of the components of the base current density vs the emitter-base applied voltage for an InAlAs/InGaAs HBT.

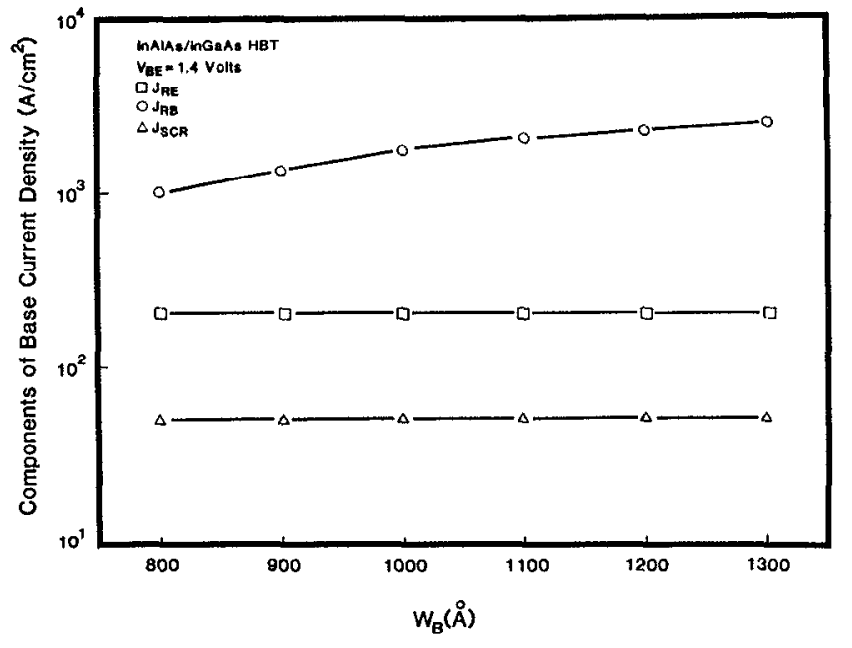

FIG. 6. Comparison of the components of the base current density vs the base width for an InAlAs/InGaAs HBT. 


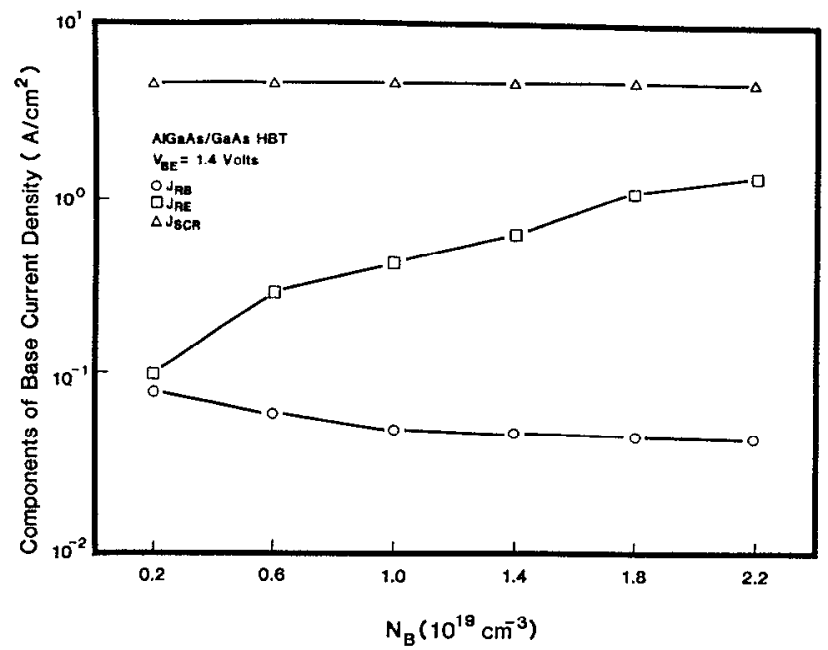

FIG. 7. Comparison of the components of the base current density vs the base doping density for an AlGaAs/GaAs HBT.

such a bias condition, neither of which was accounted for in the model. On the other hand, the model underestimates the total base current density of the InAlAs/InGaAs HBT by at most a factor of about 4 as the voltage becomes small. This is perhaps due to the fact that the surface recombination current is neglected by our analysis, which, as discussed in Sec. II, is adequate for a HBT with a base width less than or equal to about $1000 \AA$ like the device in Fig. 15 but is questionable for the HBT in Fig. 16 which has a base width of $2000 \AA$. The overall disagreement shown in Fig. 16 may also result from the deviation of the material and process parameters used in our calculations (listed in Table II). The present model does predict a trend that agrees reasonably well with experimental results for both devices. Thus the model permits a degree of insight into the influence of various device parameters on HBT base current performance and allows an estimate into the relative im-

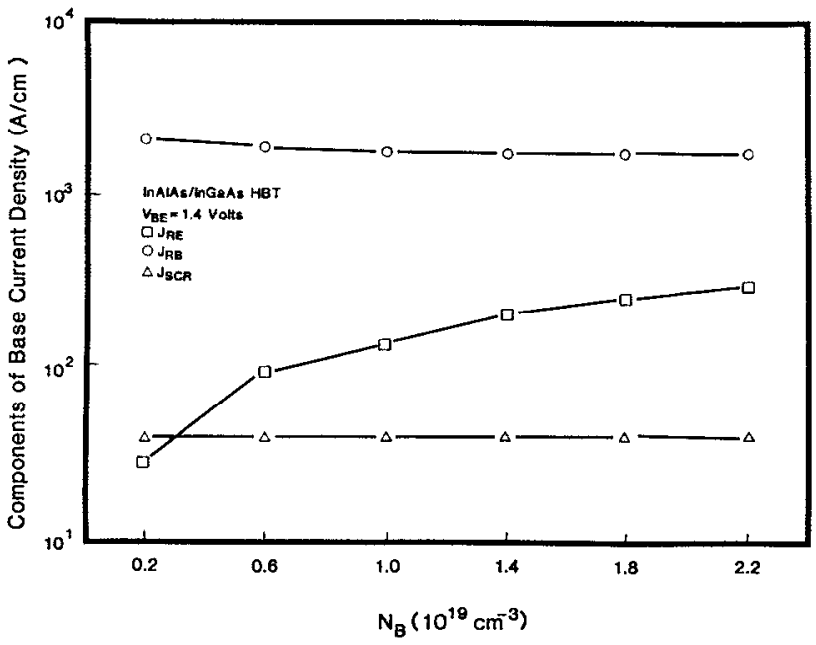

FIG. 8. Comparison of the components of the base current density vs the base doping density for an InAlAs/InGaAs HBT.

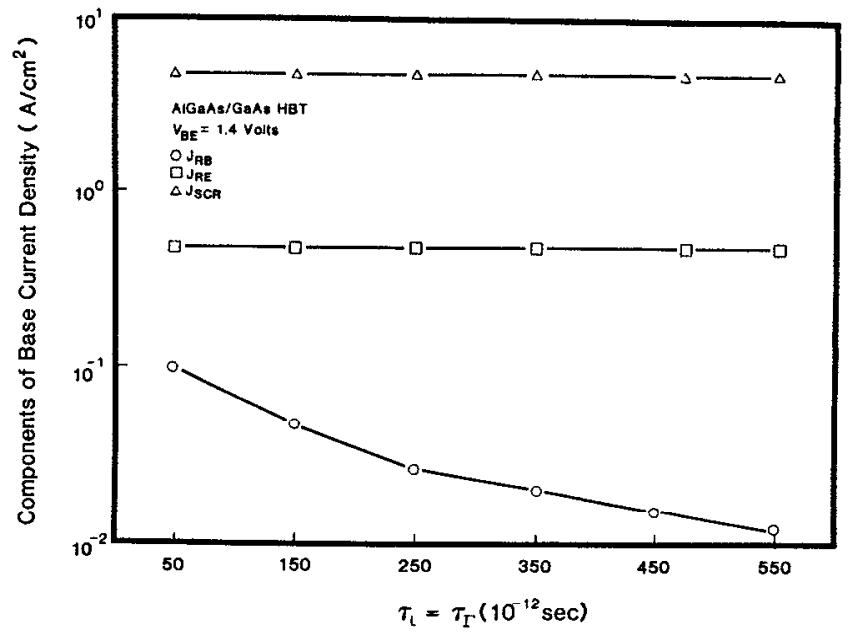

FIG. 9. Comparison of the components of the base current density vs the base minority-carrier lifetime for an AlGaAs/GaAs HBT.

portance of the base current components in $\mathrm{AlGaAs} / \mathrm{GaAs}$ and InAlAs/InGaAs HBTs.

\section{CONCLUSION}

The following conclusions can be made.

(i) Depending on $N_{t I}$ and on the bias condition, either $J_{\mathrm{SCR}}$ or $J_{\mathrm{RE}}$ is the dominant $J_{B}$ component for AlGaAs/ GaAs HBTs and either $J_{\mathrm{SCR}}$ or $J_{\mathrm{RB}}$ is the dominant $J_{B}$ component for InAlAs/InGaAs HBTs.

(ii) For typical AlGaAs/GaAs and InAlAs/InGaAs HBTs, $J_{\mathrm{SCR}}$ dominates $J_{B}$ when the emitter-base junction is not highly forward biased. As the voltage increases, however, $J_{\mathrm{RE}}$ and $J_{\mathrm{RB}}$ become important for AlGaAs/GaAs and InAlAs/InGaAs HBTs, respectively.

(iii) $J_{B}$ components are relatively insensitive to the variations of base doping concentration, base layer thickness, and base minority-carrier lifetime.

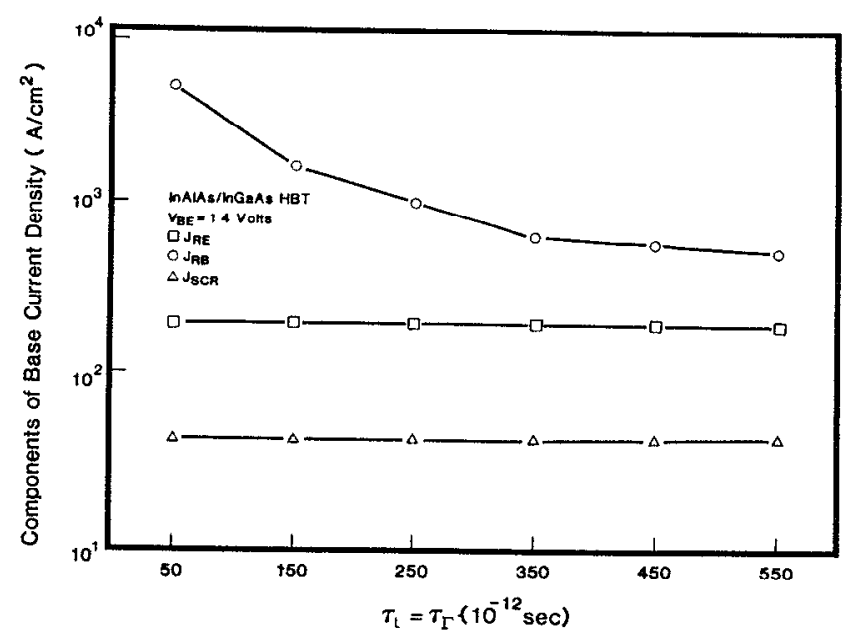

FIG. 10. Comparison of the components of the base current density vs the base minority-carrier lifetime for an InAlAs/InGaAs HBT. 


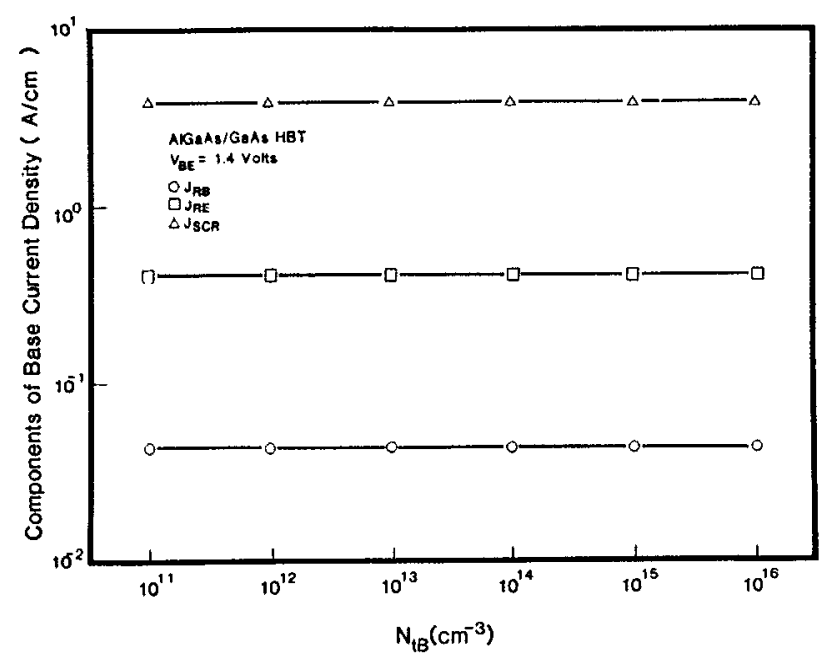

FIG. 11. Comparison of the components of the base current density vs the bulk space-charge-region trapping density for an AlGaAs/GaAs HBT.

(iv) $J_{\mathrm{SCR}}$ depends very strongly on $N_{t I}$ but very weakly on $N_{t B}$ but very weakly on $N_{t B}$, suggesting that the total base current, and thus the current gain, is influenced heavily by $N_{t l}$. This indicates that care should be taken during device fabrication so that lattice mismatch between emitter and base, which causes $N_{t I}$, is minimized. On the other hand, one does not need to worry too much about deep-level traps in the bulk emitter-base space-charge region, which yields $N_{t B}$, introduced predominantly by the diffusion of impurity dopant (e.g., Be) from the $p$-type base into the $n$-type emitter. ${ }^{25}$

(v) Increasing $N_{E}$, which reduces $J_{\mathrm{RE}}$, will reduce the total base current and thus increase the current gain in typical AlGaAs/GaAs HBTs if $V_{\mathrm{BE}}$ is large, but not otherwise. Also, increasing $N_{E}$ will not benefit the current gain in InAlAs/InGaAs HBTs because $J_{\mathrm{RE}}$ is relatively unimportant in such devices.

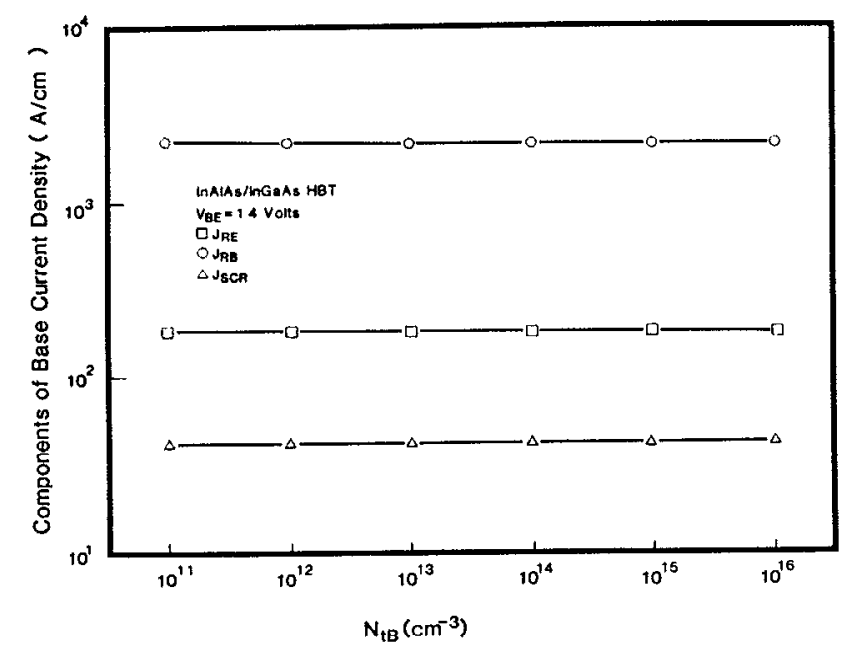

FIG. 12. Comparison of the components of the base current density vs the bulk space-charge-region trapping density for an InAlAs/InGaAs HBT.

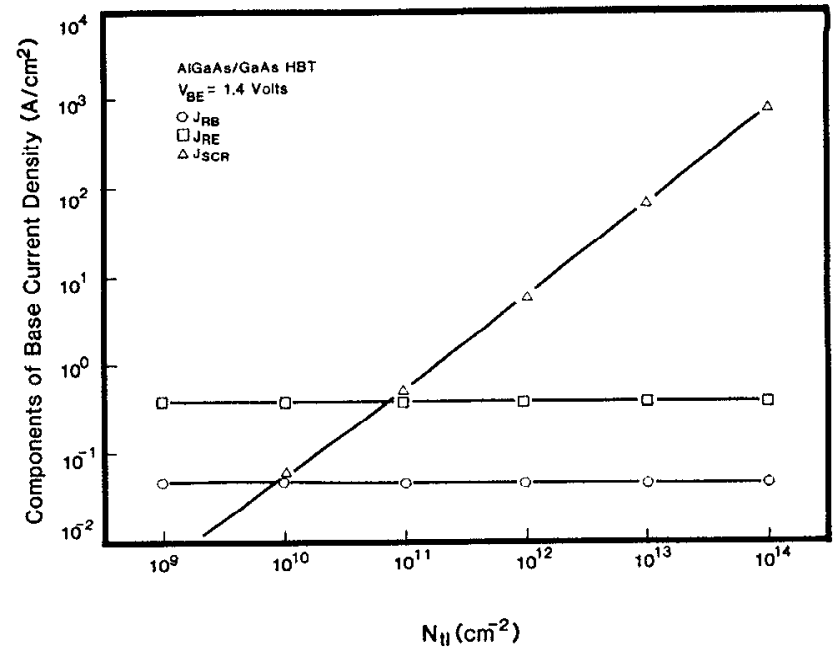

FIG. 13. Comparison of the components of the base current density vs the heterointerface state density for an AlGaAs/GaAs HBT.

(vi) For a HBT with a graded base, the charge transport in the base is enhanced by the builtin field, which reduces $J_{\mathrm{RB}}$. Also, base grading does not affect $J_{\mathrm{SCR}}$ and $J_{\mathrm{RE}}$. Therefore, at a typical operation voltage $V_{\mathrm{BE}} \approx 1.3-1.4 \mathrm{~V}$, base grading will not improve significantly the current gain of a typical AlGaAs/GaAs HBT but will improve considerably the current gain of a typical InAlAs/InGaAs HBT.

(vii) A numerical study ${ }^{4}$ and experimental observation ${ }^{26}$ have found that the elimination of the spike by grading the emitter-base heterojunction leads to an increase in $J_{n}\left(X_{2}\right)$, and thus $J_{\mathrm{RB}}$. Also, heterojunction grading increases electron-hole recombination in the emitter-base space-charge region, ${ }^{4}$ which increases $J_{\mathrm{SCR}}$, but results in a larger emitter-base valence-band discontinuity, ${ }^{27}$ which reduces $J_{\mathrm{RE}}$. Thus $J_{\mathrm{SCR}}$ is still the dominant component of base current density for both AlGaAs/GaAs and InAlAs/

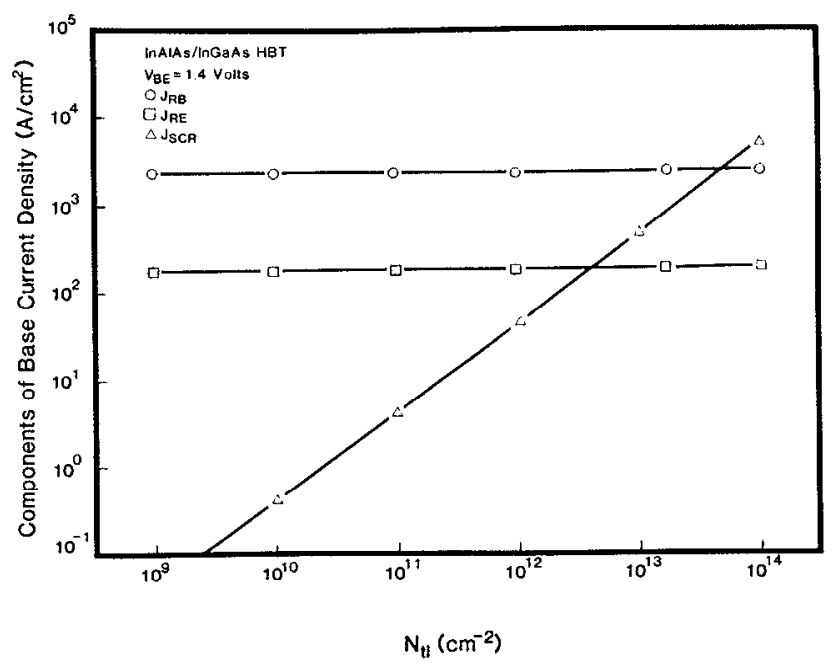

FIG. 14. Comparison of the components of the base current density vs the heterointerface state density for an InAlAs/GaAs HBT. 


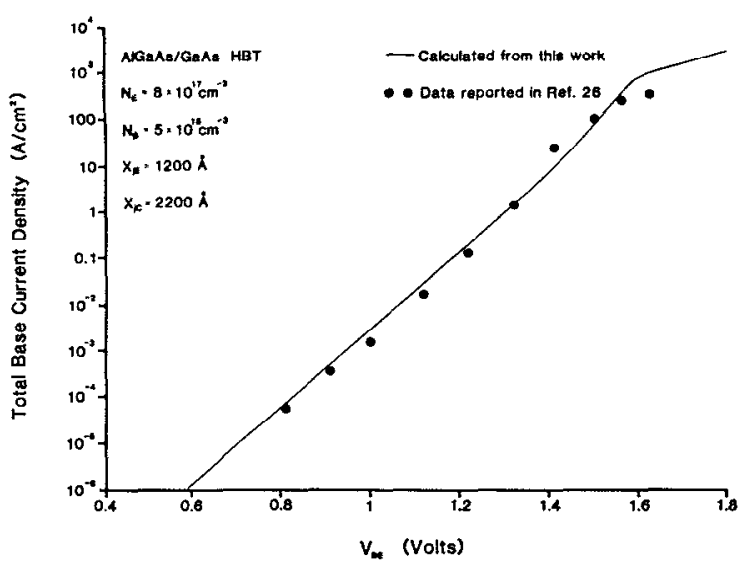

FIG. 15. Dependence of total base current on emitter-base voltage calculated from the present model and from data reported in Ref. 26 for an AlGaAs/GaAs HB'.

InGaAs HBTs with heterojunction grading when they operate at small and medium voltages. For higher voltages, either $J_{\mathrm{SCR}}$ or $J_{\mathrm{RB}}$ will become the dominant component depending on the degree of grading which affects the degree of increase in $J_{\mathrm{SCR}}$ and $J_{\mathrm{RB}}$.

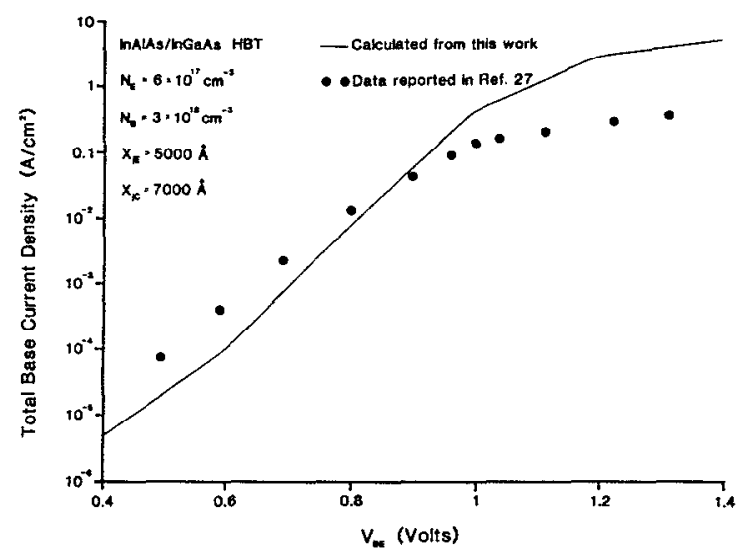

FIG. 16. Dependence of total base current on emitter-base voltage calculated from the present model and from data reported in Ref. 27 for an InAlAs/InGaAs HBT. (viii) InAlAs/InGaAs HBTs are sometimes operated at a lower $V_{\mathrm{BE}}$ (about $0.8 \mathrm{~V}$ ) than the typical HBT operation voltage of $1.4 \mathrm{~V}$ due to the much smaller band gap of InGaAs. For this bias condition, $J_{\mathrm{SCR}}$ is the dominant component in $J_{B}$.

\section{ACKNOWLEDGMENT}

This work was supported in part by the Defense Advanced Research Project Agency under Contract No. 6502-719.

${ }^{1}$ P. M. Asbeck, IEEE Trans. Electron Devices ED-34, 2571 (1987).

${ }^{2}$ H. Kroemer, Proc. IEEE 70, 13 (1982).

${ }^{3}$ M. Shur, GaAs Devices and Circuits (Plenum, New York, 1986).

${ }^{4}$ A. Das and M. S. Lundstrom, IEEE Trans. Electron Devices ED-35, 863 (1988).

${ }^{5}$ R. Katoh, M. Kurata, and J. Yashida, IEEE Trans. Electron Devices ED-36, 846 (1989).

'J. J. Liou, W. W. Wong, and J. S. Yuan, Solid-State Electron. 33, 845 (1990).

${ }^{7}$ A. Furukawa, K. Ohta, and T. Baba, IEEE IEDM Washington, DC, 615 (1987).

${ }^{8}$ Y. S. Hiraoka and J. Yoshida, IEEE Trans. Electron Devices ED-35, 857 (1988).

${ }^{9}$ H.-H. Lin and S.-C. Lee, Appl. Phys. Lett. 47, 839 (1985).

${ }^{10}$ P. I. Rockett, IEEE Trans. Electron Devices 35, 1573 (1988).

${ }^{11}$ R. Katoh and M. Kurata, IEEE Trans. Electron Devices 36, 2122 (1989).

${ }^{12}$ A. Chatterjee and A. H. Marshak, Solid-State Electron. 24, 1111 (1981).

${ }^{13}$ M. S. Lundstrom and R. J. Schuelke, Solid-State Electron. 25, 683 (1982).

${ }^{14}$ S. M. Sze, Physics of Semiconductor Devices, 2nd ed. (Wiley, New York, 1981).

${ }^{15}$ A. G. Milnes, Solid-State Electron. 29, 99 (1986).

${ }^{16} \mathrm{~W}$. Shockley and W. T. Read, Phys. Rev. 87, 835 (1952).

${ }^{17}$ E. S. Yang, Microelectronic Devices, (McGraw-Hill, New York, 1988).

${ }^{18}$ M. S. Shur, IEEE Trans. Electron Devices ED-35, 1564 (1988).

${ }^{19}$ J. J. Liou, IEEE Trans. Electron Devices ED-36, 1850 (1989).

${ }^{20}$ S. Adachi, J. Appl. Phys. 58, R1 (1985).

${ }^{21}$ S. Adachi, J. Appl. Phys. 53, 8775 (1982).

${ }^{22}$ H. Kroemer and W. Y. Chien, Appl. Phys. Lett. 33, 749 (1978).

${ }^{23}$ S. Tiwari and D. J. Frank, IEEE Trans. Electron Devices 36, 2105 (1989)

${ }^{24}$ W. Lee and C. G. Fonstad, IEEE Electron Device Lett. EDL-7, 683 (1986).

${ }^{25}$ T. S. Low and D. E. Mars, Appl. Phys. Lett. 55, 2423 (1989).

${ }^{26}$ R. Fischer, T. Henderson, J. Klem, N. Chand, and H. Morkoc, Solid State Electron. 29, 193 (1986).

${ }^{27}$ F. Capasso, in The Physics of Submicron Semiconductor Devices, edited by H. L. Grubin, D. K. Ferry, and C. Jacoboni (Plenum, New York, 1988). 